\title{
Megakaryocytes compensate for Kit insufficiency in murine arthritis
}

\author{
Pierre Cunin, ${ }^{1}$ Loka R. Penke, ${ }^{1}$ Jonathan N. Thon, ${ }^{2}$ Paul A. Monach, ${ }^{3}$ Tatiana Jones, ${ }^{4}$ Margaret H. Chang, ${ }^{1,5}$ Mary M. Chen, ${ }^{1}$ \\ Imene Melki, ${ }^{6}$ Steve Lacroix, ${ }^{7}$ Yoichiro Iwakura, ${ }^{8}$ Jerry Ware, ${ }^{9}$ Michael F. Gurish, ${ }^{1}$ Joseph E. Italiano, ${ }^{2,10}$ \\ Eric Boilard, ${ }^{6}$ and Peter A. Nigrovic ${ }^{1,5}$

\begin{abstract}
'Department of Medicine, Division of Rheumatology, Immunology and Allergy, ${ }^{2}$ Department of Medicine, Hematology Division, Brigham and Women's Hospital, Harvard Medical School, Boston, Massachusetts, USA. ${ }^{3}$ Department of Medicine, Section of Rheumatology, Boston University School of Medicine, Boston, Massachusetts, USA. ${ }^{2}$ Department of Clinical Laboratory and Nutritional Science, University of Massachusetts Lowell, Lowell, Massachusetts, USA. 5DDepartment of Medicine, Division of Immunology, Boston Children's Hospital, Harvard Medical School, Boston, Massachusetts, USA. ${ }^{6}$ Centre de Recherche du Centre Hospitalier Universitaire de Québec (CHUL), and Département de Microbiologie et Infectiologie, Faculté de Médecine de l'Université Laval, Québec, Québec, Canada. ${ }^{7} \mathrm{CHUL}$ and Département de Médecine Moléculaire-Université Laval, Faculté de Médecine de l'Université Laval, Québec, Quebec, Canada. ${ }^{8}$ Center for Animal Disease Models, Research Institute for Biomedical Sciences, Tokyo University of Sciences, Chiba, Japan. ${ }^{9}$ Department of Physiology and Biophysics, University of Arkansas for Medical Sciences, Little Rock, Arkansas, USA. ${ }^{10}$ Vascular Biology
\end{abstract} \\ Program, Department of Surgery, Boston Children's Hospital, Harvard Medical School, Boston, Massachusetts, USA
}

The growth factor receptor Kit is involved in hematopoietic and nonhematopoietic development. Mice bearing Kit defects lack mast cells; however, strains bearing different Kit alleles exhibit diverse phenotypes. Herein, we investigated factors underlying differential sensitivity to IgG-mediated arthritis in 2 mast cell-deficient murine lines: Kit ${ }^{\text {wh }}{ }^{\text {wsh }}$, which develops

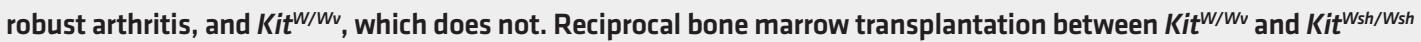
mice revealed that arthritis resistance reflects a hematopoietic defect in addition to mast cell deficiency. In Kit ${ }^{W / W_{v}}$ mice, restoration of susceptibility to IgG-mediated arthritis was neutrophil independent but required IL-1 and the platelet/ megakaryocyte markers NF-E2 and glycoprotein VI. In Kit ${ }^{W / W_{v}}$ mice, platelets were present in numbers similar to those in WT animals and functionally intact, and transfer of WT platelets did not restore arthritis susceptibility. These data implicated a platelet-independent role for the megakaryocyte, a Kit-dependent lineage that is selectively deficient in Kit ${ }^{W^{w} W_{v}}$ mice. Megakaryocytes secreted IL-1 directly and as a component of circulating microparticles, which activated synovial fibroblasts in an IL-1-dependent manner. Transfer of WT but not IL-1-deficient megakaryocytes restored arthritis susceptibility to Kit ${ }^{W / W_{v}}$ mice. These findings identify functional redundancy among Kit-dependent hematopoietic lineages and establish an unanticipated capacity of megakaryocytes to mediate IL-1-driven systemic inflammatory disease.

\section{Introduction}

Mast cells (MCs) were first described as tissue-resident cells that stain brightly with aniline dyes (1). Their highly granular appearance reflects their capacity to release a wide range of preformed mediators, including proteases, heparin, and cytokines. MCs express multiple activating receptors and predate the evolution of humoral immunity, suggesting substantial importance to the survival of the organism (2).

For identifying and characterizing these in vivo functions, the field of MC biology has been aided by the availability of mice that lack this lineage. Historically, most studies have employed mice with mutations affecting the MC growth factor KitL (also called stem cell factor) or its receptor Kit. For example, Kit ${ }^{W / W v}(\mathrm{~W} / \mathrm{Wv})$

Conflict of interest: J.N. Thon and J.E. Italiano are founders of Platelet BioGenesis. M.M. Chen is an employee of Takeda Pharmaceuticals. M.F. Gurish has served as a consultant for Karos Pharmaceuticals. P.A. Nigrovic receives salary support as a member of the steering committee of the Childhood Arthritis and Rheumatology Research Alliance, authorship royalties from UpToDate Inc. and the American Academy of Pediatrics, and holds investigator-initiated grants from Novartis and Sobi.

Submitted: September 23, 2015; Accepted: February 2, 2017

Reference information: / Clin Invest. 2017;127(5):1714-1724

https://doi.org/10.1172/JCI84598. mice express hypofunctional Kit, while $\mathrm{Kit}^{\mathrm{Wsh} / \mathrm{Wsh}}$ (Wsh) mice have a genetic inversion upstream of Kit that alters its expression in MCs, and $K i t^{\text {sl/sld }}$ (Sl/Sld) mice lack surface-bound KitL (3). However, the $\mathrm{Kit} / \mathrm{KitL}$ axis is relevant for many lineages, and these MC-deficient animals exhibit broad phenotypic abnormalities. W/Wv mice are anemic, sterile, megakaryocytopenic, and neutropenic, while Wsh mice display an excess of neutrophils, megakaryocytes (MKs), and platelets $(4,5)$. To identify the specific contribution of MCs, Galli and colleagues developed a system for adoptive transfer of MCs into $\mathrm{W} / \mathrm{Wv}$ mice (6). Reconstitution is neither complete nor fully physiologic in distribution. However, correction of an in vivo phenotype by this so-called "MC knockin" approach has been considered strong evidence for a (patho)physiological role for the MC. Such studies form the bedrock of our understanding of in vivo MC biology.

Recently, conclusions from these foundational studies have been unsettled by work using newly engineered MC-deficient animals $(7,8)$. For example, in experimental contact allergy, studies with mice susceptible to inducible MC depletion found that these cells promote pathology, whereas MC reconstitution had indicated a suppressive role (8-10). Similar controversy has arisen with regard to antibody-mediated arthritis. Both $\mathrm{W} / \mathrm{Wv}$ and $\mathrm{Sl} / \mathrm{Sld}$ mice are resistant to arthritis induced by transfer of IgG autoanti- 

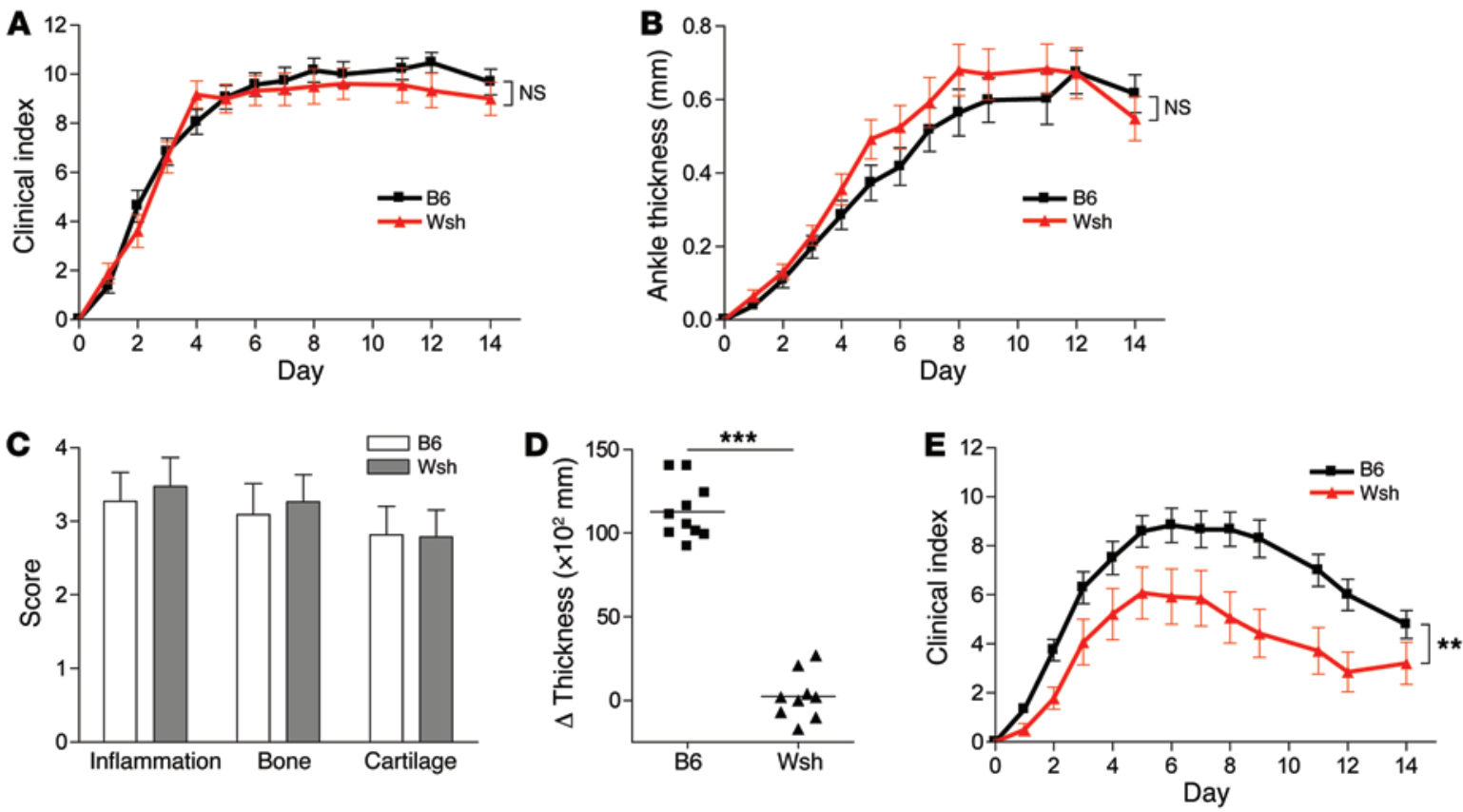

Figure 1. Dose-dependent susceptibility of Wsh mice to K/BxN serum transfer arthritis. (A-D) Male B6 $(n=19)$ and Wsh $(n=18)$ mice were treated with 150 $\mu$ i.p. K/BxN serum on days 0 and 2 and assessed for arthritis over 2 weeks. Results were pooled from 4 identical experiments using a single pooled batch of $\mathrm{K} / \mathrm{BxN}$ serum. (A) Clinical scoring on a 0 to 12 scale $(P=\mathrm{NS})$. (B) Ankle thickness change $(P=\mathrm{NS})$. (C) Histological scoring on ankle sections (19 to 22 ankles/ group, all comparisons $P=N S$ ). (D) Acute increase in ankle and wrist thickness (flare) assessed 30 minutes after injection of $\mathrm{K} / \mathrm{BxN}$ serum (assessed in $n=$ 10/group, $P<0.0001$ ). (E) $50 \mu \mathrm{l} \mathrm{i.p.} \mathrm{K/BxN} \mathrm{serum} \mathrm{on} \mathrm{days} 0$ and 2 (B6, $n=24 ;$ Wsh, $n=14$ pooled from 4 experiments; $P=0.0088$ ). ${ }^{* *} P<0.01,{ }^{* * *} P<0.001$.

body-containing serum from $\mathrm{K} / \mathrm{BxN}$ mice $(11,12)$, a finding reproduced in Pretty2 mice bearing a point mutation in the Kit kinase domain (13). Resistance extends to collagen antibody-induced arthritis and is overcome by MC engraftment $(11,13,14)$. However, Wsh mice are not resistant to arthritis $(14,15)$. Further, mice congenitally lacking all MCs through genotoxicity resulting from MC-specific overexpression of Cre (carboxypeptidase A3-Cre, termed Cre-Master mice) as well as mice largely deficient in connective tissue MCs develop K/BxN arthritis normally $(7,16)$. These findings have been interpreted as evidence that Kit mutants may not accurately reflect the in vivo role of MCs. For example, apparent arthritis dependence on MCs in $\mathrm{W} / \mathrm{Wv}$ has been proposed to reflect concomitant neutropenia, though this hypothesis has never been tested experimentally $(3,7,14)$.

However, not all support for a role of MCs in arthritis derives from Kit mutants. Mice lacking the highly MC-specific proteases mMCP6 or mMCP7 show impaired arthritis, a phenotype exposed only at submaximal doses of $\mathrm{K} / \mathrm{BxN}$ serum $(17,18)$. These results suggest that the contribution of MCs to arthritis is not "all or none," but instead that understanding their contribution requires careful experimental dissection of the context in which MCs play, or fail to play, a pathogenic role.

We therefore sought to understand the basis for differential susceptibility to $\mathrm{K} / \mathrm{BxN}$ arthritis in W/Wv and Wsh mice. We found that $\mathrm{MC}$ dependence in $\mathrm{W} / \mathrm{Wv}$ mice reflected both the arthritisresistant radioresistant background and the failure of other hematopoietic lineages to compensate for MC deficiency. Unexpectedly, as assessed by both genetic and engraftment approaches, the deficient hematopoietic lineage was not the neutrophil, but instead the MK, which also depends on Kit for normal develop- ment. MKs proved to be a sufficient source for IL-1 in arthritis, independent of platelets and potentially mediated through release of IL-1-rich MK microparticles into the systemic circulation.

\section{Results}

Wsh mice exhibit relative arthritis resistance at low serum dose. Consistent with published data, Wsh mice exhibited full susceptibility to $\mathrm{K} / \mathrm{BxN}$ serum transfer arthritis at conventional doses (150 $\mu \mathrm{l}$ serum i.p. on days 0 and 2), as assessed by clinical scoring and change in paw thickness (Figure 1, A and B). Histological scores disclosed no difference in inflammation, bone erosion, or cartilage injury (Figure 1C). Normal arthritis evolved despite absence of immune complex-mediated vascular leak as assessed by paw edema, confirming a nonredundant role for MCs in this phenotype (Figure 1D) $(19,20)$.

These results appear to contradict data from MC knockin experiments. We therefore considered whether bone marrowderived MC (BMMC) engraftment might be contaminated by lineages beyond the $\mathrm{MC}$, leading to incorrect attribution of effect. However, no "off target" engraftment could be identified 10 weeks following transfer of CD45.1-expressing BMMCs into recipient CD $45.2 \mathrm{~W} / \mathrm{Wv}$ mice. Engrafted mice exhibited no changes in circulating leukocyte or platelet counts, and baseline anemia failed to correct (Supplemental Figure 1A; supplemental material available online with this article; https://doi.org/10.1172/ JCI84598DS1). No circulating CD45.1 $1^{+}$cells could be detected, and $\mathrm{CD} 45.1^{+}$cells in bone marrow and spleen were restricted to Fc\&R1 ${ }^{+}$Kit $^{\text {hi }}$ MCs (Supplemental Figure 1B).

We then considered the possibility that, as observed in mMCP6- and mMCP7-deficient mice, Wsh animals might exhib- 
A

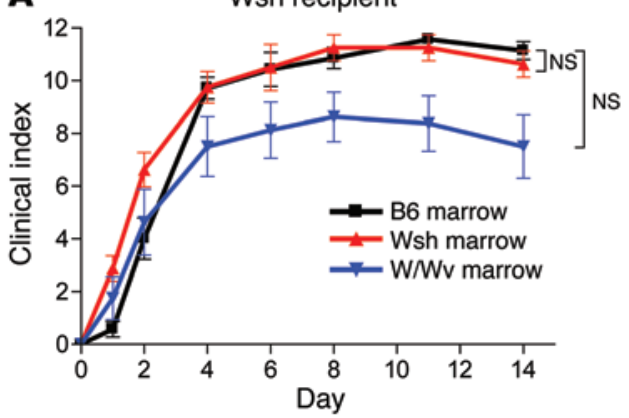

B

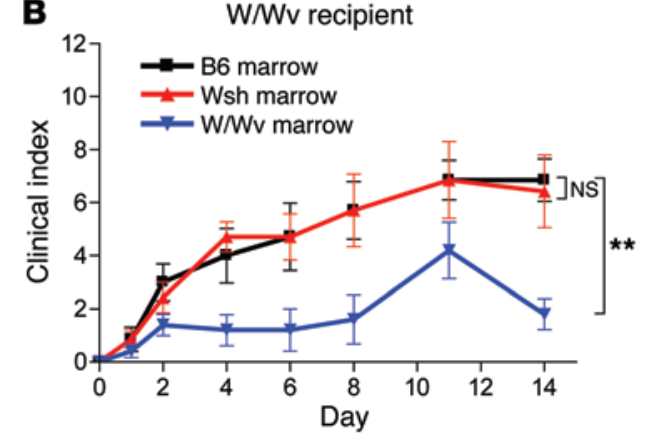

Figure 2. Mixed hematopoietic and radioresistant contributions to differential arthritis susceptibility in Wsh and W/Wv mice. (A and B) Wsh and W/Wv mice were lethally irradiated and engrafted for 10 weeks with whole donor marrow from W/Wv, Wsh, or B6 donors, followed by initiation of arthritis with $150 \mu \mathrm{l}$ i.p. K/BxN serum on days 0 and 2 ( $n=5-8$ mice/group pooled from 2 experiments; W/Wv arms, $P=0.0096$ ). B6 recipient data are in Supplemental Figure 4C. ${ }^{*} P<0.01$. (A) B6 vs. Wsh: $P=N S$; B6 vs. W/Wv: $P=N S$; Wsh vs W/Wv: $P=0.0410$.

(B) B6 vs. Wsh: $P=\mathrm{NS}$; B6 vs. W/Wv: $P=0.0096$; Wsh vs W/Wv: $P=0.0155$.

it reduced arthritis susceptibility at a lower dose of $\mathrm{K} / \mathrm{BxN}$ serum. Indeed, such susceptibility emerged (Figure 1E). Whereas i.v. transfer of BMMCs into Wsh mice resulted in no detectable engraftment in joint tissue (triplicate experiments, $n=27$ engrafted Wsh mice, data not shown), we could not employ MC knockin to test whether impaired susceptibility reflected MC deficiency alone. Of note, $\mathrm{K} / \mathrm{BxN}$ serum titration in MC-deficient Cre-Master mice failed to identify dose-dependent susceptibility (Supplemental Figure 2, A and B). These findings suggest that the basis for dose-dependent partial disease resistance in Wsh is likely complex.

Susceptibility of Wh mice does not reflect an intrinsic inflammatory phenotype. Hematopoietic aberrancy associated with the Wsh genetic inversion includes neutrophilia, thrombocytosis, and splenomegaly $(5,14)$. This phenotype is reminiscent of a systemic inflammatory state that might "bypass" the requirement for MCs in the way that administration of IL- $1 \beta$ enables arthritis in $\mathrm{W} / \mathrm{Wv}$ mice (15). Consistent with this possibility, administration of LPS mimicked administration of IL-1 $\beta$ in rendering $\mathrm{W} / \mathrm{Wv}$ animals susceptible to arthritis (Supplemental Figure 3A). However, the inflammatory markers $\mathrm{C}$-reactive protein (CRP) and serum amyloid A were not elevated in unmanipulated Wsh mice, nor could susceptible C57BL/6 (B6) back- we identify unusual circulating levels of IL- $1 \beta$ and IL- 6 at baseline or after administration of $\mathrm{K} / \mathrm{BxN}$ serum (Supplemental Figure 3, B and C). Further, splenectomy failed to alter arthritis in Wsh mice (Supplemental Figure 3D). We conclude that an aberrant baseline inflammatory state does not mediate Wsh arthritis susceptibility.

Role for radioresistant and hematopoietic factors in $W / W v$ arthritis resistance. The Wsh mutation arose spontaneously during a $\mathrm{C} 3 \mathrm{H} / \mathrm{HeH} \times 101 / \mathrm{H}$ cross (21), but has been bred into the arthritisritis at conventional serum doses typically achieves a mean clinical index of approximately 10 out of 12 (see Figure 1A). In contrast, $\mathrm{W} / \mathrm{Wv}$ mice have a mixed $\mathrm{WB} \times \mathrm{B} 6$ background (termed WBB6). WBB6 mice without Kit mutations exhibit reduced arthritis intensity, usually no higher than 6 out of 12 $(11,15)$. We sought to test the role of radioresistant and hematopoietic elements in differential arthritis susceptibility using criss-cross bone marrow transplantation.

$\mathrm{W} / \mathrm{Wv}$ marrow enabled arthritis in both Wsh and B6 mice, although disease intensity was somewhat reduced (Figure 2A; Supplemental Figure 4A; confirmation of engraftment, Supplemental Figure 4, B and C). B6 marrow restored arthritis susceptibility in $\mathrm{W} / \mathrm{Wv}$ mice, although again disease intensity was reduced, remaining at the level of that seen in the WBB6 background (Figure 2B). These observations implicate both radioresistant and hematopoietic factors in $\mathrm{W} / \mathrm{Wv}$ arthritis resistance. Surprisingly, despite the inability of Wsh mice to generate MCs, Wsh marrow also restored arthritis in W/Wv mice (Figure 2B). This result demonstrates that cells other than MCs and produced by either $\mathrm{B} 6$ or Wsh marrow can overcome arthritis resistance in $\mathrm{W} / \mathrm{Wv}$ mice.
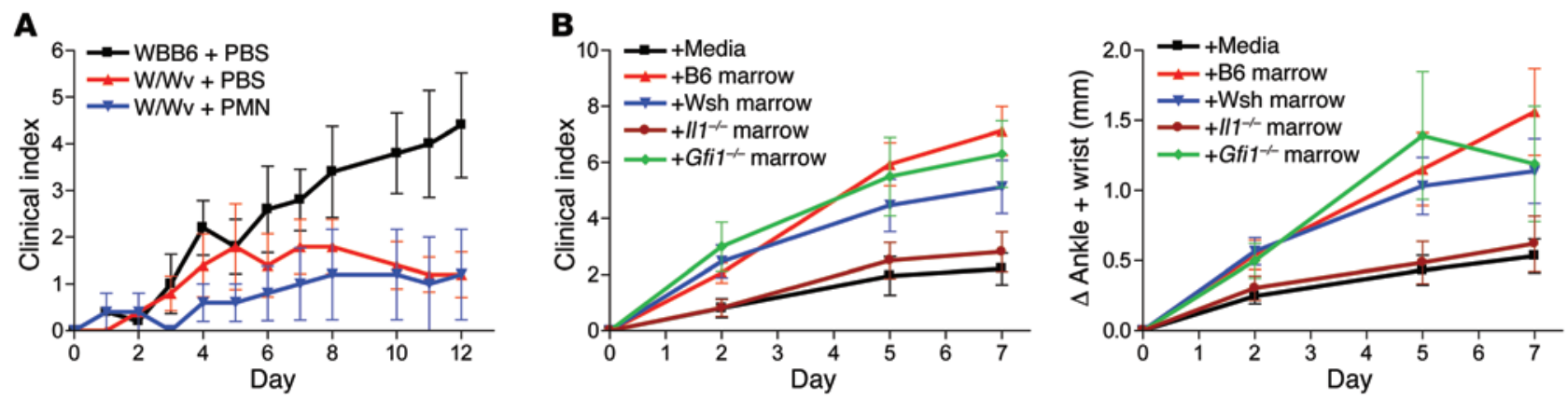

Figure 3. A nonneutrophil IL-1-expressing marrow lineage bypasses the requirement for MC in W/Wv mice. (A) W/Wv mice were injected i.v. with purified B6 bone marrow neutrophils ( 2 donors/d on days $0-4$ ) or saline control as well as $150 \mu \mathrm{l}$ i.p. K/BxN serum on days 0 and 2 ( $n=5 /$ group; 1 of 2 similar experiments shown). (B) W/Wv mice received whole B6, Wsh, G fi $^{-1 /}$, or $1 / 1^{-1-}$ bone marrow by tail vein (1 donor per 2 recipients), and arthritis was initiated 14 days later as above. Left, clinical scoring (mock engrafted vs. B6 engrafted, $P=0.0001$; mock engrafted vs. Wsh engrafted, $P=0.0117$; mock engrafted vs. G fi $11^{-/-}$engrafted, $P=0.004$; mock engrafted vs. $I / 1^{-/-}$engrafted, $P=\mathrm{NS}$ ). Right, ankle and wrist thickness change (mock engrafted vs. B6 engrafted, $P=0.012$; mock engrafted vs. Wsh engrafted, $P=0.03$; mock engrafted vs. Gfi ${ }^{-/-}$engrafted, $P=0.038$; mock engrafted vs. $I 11^{-/-}$engrafted, $P=\mathrm{NS}$ ). Mock engrafted, $n=20$; B6 engrafted, $n=16$; Wsh engrafted, $n=15$; Gfi ${ }^{-/-}$engrafted, $n=10$; $I 11^{-1-}$ engrafted, $n=16$; pool of 2-3 experiments. 


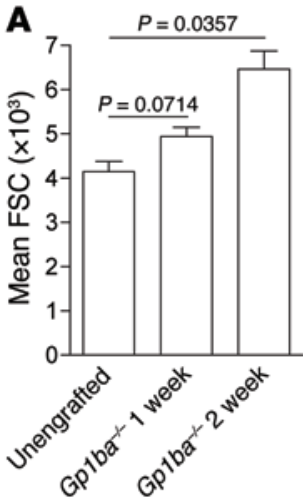

B
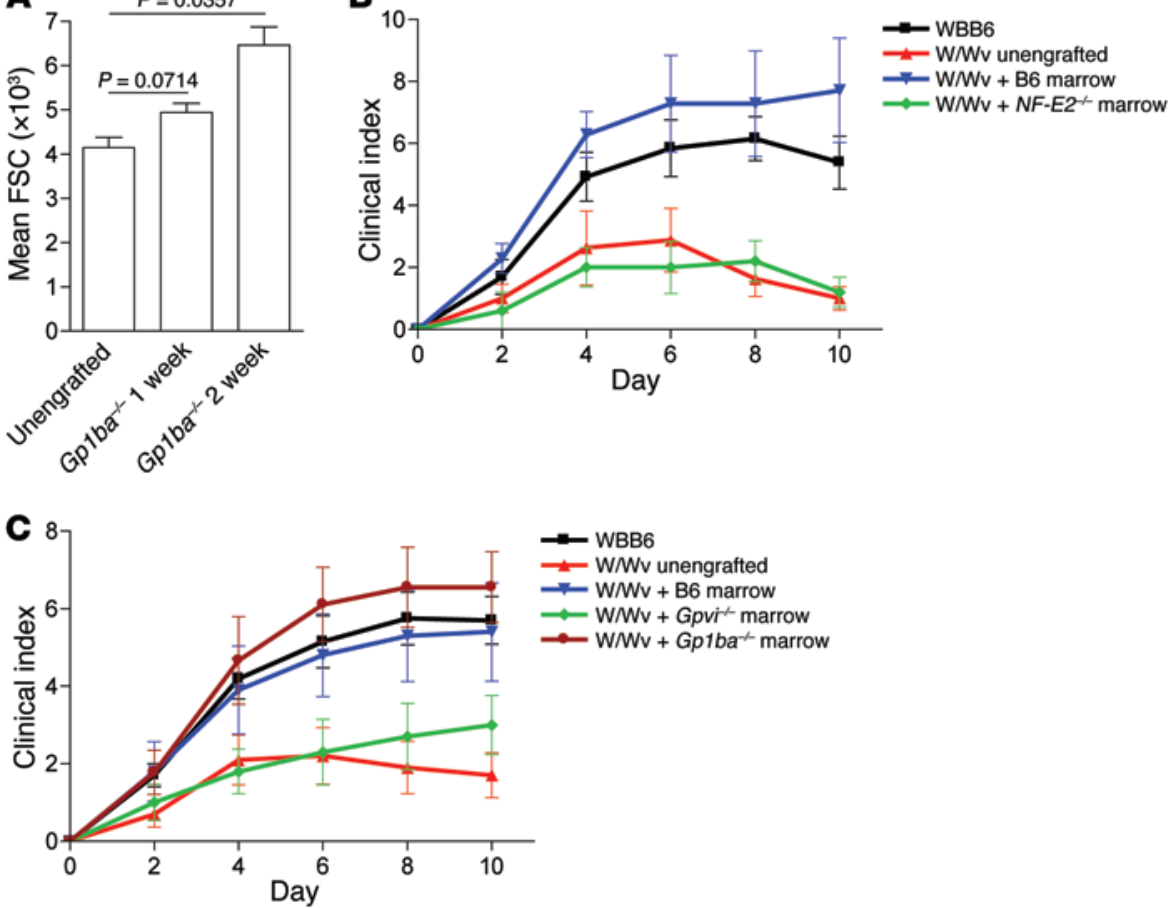

Figure 4. The platelet/MK lineage mediates arthritis restoration in W/Wv mice. (A) W/Wv mice were engrafted with whole $G \mathrm{p}^{\mathrm{b}} \mathrm{ba}^{-/}$bone marrow (1 donor per 2 recipients) and platelet size (forward scatter [FSC]) monitored by flow cytometry at 1 and 2 weeks ( $n=3$ unengrafted and 5 recipient mice). (B and $\mathbf{C}$ ) Whole bone marrow was transferred i.v. in W/Wv mice (1 donor per 2 recipients) and arthritis initiated with $150 \mu \mathrm{l} \mathrm{K} / \mathrm{BxN}$ serum on days 0 and 2. (B) Clinical score after transfer of $\mathrm{NF}-\mathrm{E2}^{-/-}$and control B6 marrow. W/Wv vs. NF-E2 $2^{-/-}$engrafted, $P=\mathrm{NS} ; \mathrm{W} / \mathrm{Wv}$ vs. B6 engrafted, $P=0.006$. $n=8 \mathrm{~W} / \mathrm{Wv} ; n=5 \mathrm{~W} / \mathrm{Wv}+\mathrm{NF}-E 2^{-/-}(1$ fatality); $n=7 \mathrm{~W} / \mathrm{Wv}+\mathrm{B} 6$ (1 failed engraftment); $n=13$ WBB6; pooled from 2 experiments. (C) Clinical score after transfer of $G p v i^{-/-}, \mathrm{Cp} \mathrm{ba}^{-/-}$, and control B6 marrow. W/Wv vs. Gpvi-/- engrafted, $P=\mathrm{NS} ; \mathrm{W} / \mathrm{W}$ v vs. B6, $P=0.026 ; \mathrm{W} / \mathrm{Wv}$ vs. Gp1 $\mathrm{ba}^{-/-}$engrafted, $P=0.001 . n=10 \mathrm{~W} / \mathrm{Wv}$; $n=10 \mathrm{~W} / \mathrm{Wv}+\mathrm{B} 6 ; n=10 \mathrm{~W} / \mathrm{Wv}+\mathrm{Cpvi}^{-/-} ; n=9$ $\mathrm{W} / \mathrm{Wv}+\mathrm{Gp} 1 \mathrm{ba}^{-/-} ; n=20 \mathrm{WBB} 6$ pooled from 2 experiments.
The hematopoietic lineage that restores arthritis susceptibility in $\mathrm{Kit}^{W / W_{v}}$ mice produces IL-1 but is not the neutrophil. Circulating neutrophils are relatively deficient in $\mathrm{W} / \mathrm{Wv}$ mice (5). Neutrophils are a key effector lineage in $\mathrm{K} / \mathrm{BxN}$ arthritis $(22,23)$, and it is therefore plausible to suggest that neutropenia could expose a requirement for MCs in W/Wv mice, in particular because engraftment studies implicate IL- 1 as a key MC effector in $\mathrm{K} / \mathrm{BxN}$ arthritis and neutrophils also elaborate IL- 1 in this setting $(15,24)$. To test this hypothesis, we employed neutrophil transfer (25). Surprisingly, W/Wv mice receiving WT neutrophils remained indistinguishable from those receiving media alone (Figure $3 \mathrm{~A}$ ). To confirm this observation, we adapted an approach that takes advantage of the early emergence of neutrophils from donor marrow (26). Either B6 or Wsh marrow completely restored arthritis susceptibility within 2 weeks of transfer into unirradiated $\mathrm{W} / \mathrm{Wv}$ recipients, without the appearance of MCs in peripheral tissues (Figure 3B). Restoration absolutely required that donor marrow be competent to express IL-1 (Figure 3B; confirmation of engraftment, Supplemental Figure 4D). However, marrow from neutrophil-deficient (and therefore arthritis resistant) mice lacking the transcription factor growth factor independent-1 (GFI-1) $(26,27)$ proved capable of restoring arthritis (Figure $3 \mathrm{~B})$. These data suggest that neutropenia is not a critical basis for arthritis resistance in $\mathrm{W} / \mathrm{Wv}$ mice, but point instead to another marrow lineage from either B6 or Wsh donors (i.e., not MCs) that can "patch" arthritis resistance. This lineage is presumably deficient in W/Wv mice, engrafts within 2 weeks after transfer of either B6 or Wsh marrow, and must elaborate IL-1.

The platelet/MK lineage mediates restoration of arthritis susceptibility. One of the Wsh hematopoietic aberrancies is thrombocytosis, associated with a marked expansion of MKs in spleen (5). In contrast, W/Wv mice exhibit a normal circulating platelet count (5), although the number of marrow MKs is reduced 5-fold, a paradox thought to reflect hypertrophy of remaining MKs (28).
Platelets are a potentially interesting source of IL-1 because IL-1expressing platelet microparticles amplify $\mathrm{K} / \mathrm{BxN}$ arthritis (29). Generation of platelet microparticles is triggered via the collagen-laminin receptor glycoprotein VI (GPVI), expressed exclusively by platelets and their parent MKs (29). We therefore tested whether MKs could engraft in an appropriate time frame. For these studies, we employed donor marrow from mice deficient in glycoprotein $1 \mathrm{~b}$ (GP1BA), a membrane receptor without which platelets emerge distinctly larger than WT platelets (30). Indeed, $\mathrm{W} / \mathrm{Wv}$ recipients of $\mathrm{Gp} 1 \mathrm{ba}^{-/-}$marrow exhibited large platelets as soon as 1 week after marrow transfer (Figure $4 \mathrm{~A}$ ).

We then examined whether the platelet/MK lineage might be responsible for correction of arthritis. The transcription factor NF-E2 is required for normal megakaryopoiesis, and NF-E2-/mice usually die from hemorrhage in parturition (31). However, from several hundred NF-E2 het $\times$ het pups, we were able to obtain $3 \mathrm{NF}-\mathrm{E} 2^{-/}$donors. Interestingly, unirradiated $\mathrm{W} / \mathrm{Wv}$ mice receiving $\mathrm{NF}-\mathrm{E} 2^{-/-}$marrow remained free of arthritis, while those receiving B6 marrow became fully susceptible (Figure 4B). $\mathrm{NF}-\mathrm{E} 2^{-/-}$mice are as anemic as $\mathrm{W} / \mathrm{Wv}$ mice, so we were unable to employ hematocrit to assess successful engraftment, though $N F-E 2^{-/-}$DNA could be detected by PCR in recipient marrow at harvest (Supplemental Figure 4E). Given the difficulty of confirming adequate chimerism, we sought a complementary approach. Recognizing the lineage specificity of GPVI (32), we employed Gpvi ${ }^{-/-}$ marrow in our short-term engraftment system. Marrow from these animals engrafted well, as determined by correction of anemia in W/Wv mice (Supplemental Figure 4F). Nevertheless, $\mathrm{W} / \mathrm{Wv}$ recipients of $\mathrm{Gpvi}^{-/-}$marrow remained remarkably arthritis resistant, whereas arthritis susceptibility was restored by WT and $\mathrm{Gp} \mathrm{ba}^{-/}$marrow (Figure 4C). We conclude that the platelet/MK lineage compensates for Kit insufficiency, including absent MCs, in the W/Wv background. 


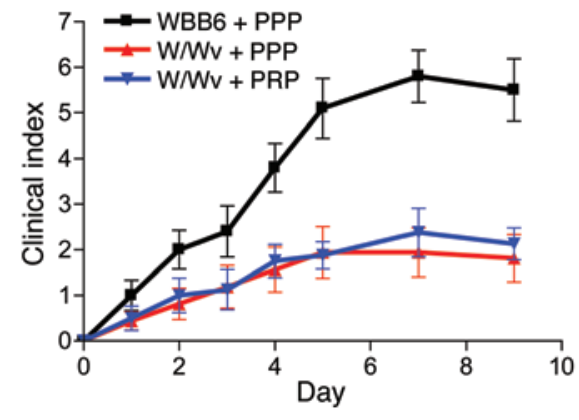

Figure 5. Platelet transfer does not restore arthritis susceptibility in W/Wv mice. Platelet-Rich Plasma (PRP) and Platelet-Poor Plasma (PPP) were purified from $\mathrm{B} 6$ donors and transferred into recipient mice i.v. (5 donors/recipient/d on days 0-4) along with $150 \mu$ i.p. K/BxN serum on days 0 and 2. PRP vs. PPP W/Wv, $P=$ NS. $n=10$ PPP-W/Wv, $n=8$ PRP$\mathrm{W} / \mathrm{Wv}, n=16 \mathrm{WBB} 6$; pooled from 2 experiments.

$B M M C$ engraftment is free of concomitant $M K$ engraftment. If MKs engraft readily from donor marrow, this raises the possibility that coengraftment of MKs might confound MC knockin, unrecognized by our CD45.1/CD45.2 system (Supplemental Figure 1), since CD45 is not expressed by platelets. We excluded this possibility in 2 ways. First, 4-week-old BMMC cultures as used for engraftment failed to yield MKs when cultured in the presence of thrombopoietin (Supplemental Figure 5A). Second, engraftment of $\mathrm{G} p 1 b a^{-/}$BMMCs into W/Wv mice did not change mean platelet size, excluding coengraftment of donor MKs (Supplemental Figure 4B).

Evidence that restoration of arthritis reflects $M K s$ rather than platelets. Given the known role of platelets in $\mathrm{K} / \mathrm{BxN}$ arthritis $(29,33)$, we hypothesized that restoration of arthritis reflected supplementation of a defective population of circulating platelets. Indeed, human platelets have been reported to express Kit, suggesting one mechanism by which the $\mathrm{W} / \mathrm{Wv}$ genetic defect might affect platelets (34). However, we could not identify Kit expression in WT murine platelets, either directly ex vivo or after stimulation with a GPVI agonist (data not shown). Further, W/Wv platelets stimulated via GPVI elaborated microparticles at least as efficiently as WBB6 platelets, microparticle concentrations of IL- $1 \alpha$ and IL-1 $\beta$ were similar, and both W/Wv and WBB6 platelet microparticles stimulated murine joint-derived fibroblast-like synoviocytes (FLS) with similar efficiency (Supplemental Figure 6, A-C). Finally, employing an established adoptive transfer system (33), transfer of WT platelets to W/Wv mice failed to restore arthritis susceptibility (Figure 5).

We therefore considered whether arthritis restoration might be mediated directly by MKs. MKs are not conventionally considered an immune lineage, although human MK lines can elaborate IL-1 and MKs are recognized as a source of circulating microparticles in humans and mice $(35,36)$. Indeed, MKs cultured from murine bone marrow elaborated IL- $1 \alpha$ and IL-1 $\beta$ and generated microparticles that contained both IL- $1 \alpha$ and IL- $1 \beta$, while nuclear IL-1 $\alpha$ was evident within a subset of mature MKs, either differentiated in vitro or from bone marrow (Figure 6, A-D). MK supernatant and MK microparticles stimulated FLS in an IL-1 receptor-dependent manner (Figure 6E). Thus, murine MKs are a potential source of systemic IL-1, even independent of their daughter platelets.
We used adoptive transfer to test whether MKs could restore arthritis susceptibility (37). We employed bone marrow MKs because they elaborate platelets more poorly than conventional fetal liver MKs (38), helping us to isolate a platelet-independent role for MKs. Control studies demonstrated that MKs tolerated i.v. transfer easily, without fragmentation or numeric loss, after passage through a 28-gauge needle (data not shown). MKs were transferred i.v. into $\mathrm{W} / \mathrm{Wv}$ mice 1 hour prior to injection of $\mathrm{K} / \mathrm{BxN}$ serum. To identify inadvertent cotransfer of other hematopoietic lineages and to track the emergence of donor platelets, some transferred MKs were derived from CD41-eYFP B6 mice (where eYFP indicates enhanced yellow fluorescent protein; ref. 39) backcrossed to express CD45.1. In duplicate experiments, MK engraftment restored arthritis susceptibility to recipient $\mathrm{W} / \mathrm{Wv}$ mice (Figure 6F). No hematopoietic coengraftment could be identified by analysis of recipient blood for CD $45.1^{+}$cells, and no MCs were detected in spleen and joint tissues (Supplemental Figure 7, A and B). To further exclude the possibility that contaminating non-MK cells were responsible for arthritis restoration, enriched MKs were magnetically sorted based on CD41 expression. Although we were able to detect some small cells in the CD41-negative fraction, we failed to detect substantial IL- $1 \alpha$ and IL-1 $\beta$ production by these cells even after LPS stimulation (Supplemental Figure 7, C and D). Moreover, when injected into $\mathrm{W} / \mathrm{Wv}$ mice, the CD41-negative fraction was unable to restore arthritis (Supplemental Figure 7E), confirming that MKs rather than a contaminating CD41negative lineage were responsible for arthritis restoration. Finally, consistent with our marrow transfer findings, MKs cultured from IL-1-deficient mice failed to restore arthritis susceptibility despite adequate engraftment as determined both by lung histology and by detection of donor-derived platelets and MK microparticles (Supplemental Figure 8).

Of note, only a very small number of eYFP-positive platelets and microparticles were observed after engraftment of CD41eYFP MKs (data not shown), consistent with very inefficient eYFP expression in these mice (39). To better assess the localization of engrafted MKs and the time course of emergent platelets and microparticles, we injected W/Wv mice with green-CMFDAlabeled MKs (where CMFDA indicates 5-chloromethylfluorescein diacetate). In agreement with published findings (37), histological evaluation 75 minutes after transfer confirmed the presence of donor MKs in lungs and to a lesser extent in spleen, but not in bone marrow or joints (Figure 7A and data not shown). Consistent with poor in vitro elaboration of platelets by bone marrow-derived MKs, donor-derived platelets made up less than $0.3 \%$ of the total platelet pool, compared with approximately $10 \%$ achieved by direct platelet transfer (33). Emergence of fluorescent platelets showed a biphasic pattern, with an early peak at 7 hours after injection and a late peak after 1 week, potentially reflecting a pool of greenCMFDA-labeled precursor MKs that matured in vivo (Figure 7B).

Aside from a small increase in size, we identified no specific in vitro abnormalities in $\mathrm{W} / \mathrm{Wv}$ MKs cultured from bone marrow (Supplemental Figure 6D). Cultured W/Wv MKs contain IL-1 $\alpha$ and IL-1 $\beta$ (Supplemental Figure 6E), they can generate microparticles (Supplemental Figure 6F), and both microparticles and supernatant from cultured W/Wv MKs activate FLS (Supplemental Figure $6 \mathrm{G}$ ). However, consistent with the in vivo phenotype (28), the 

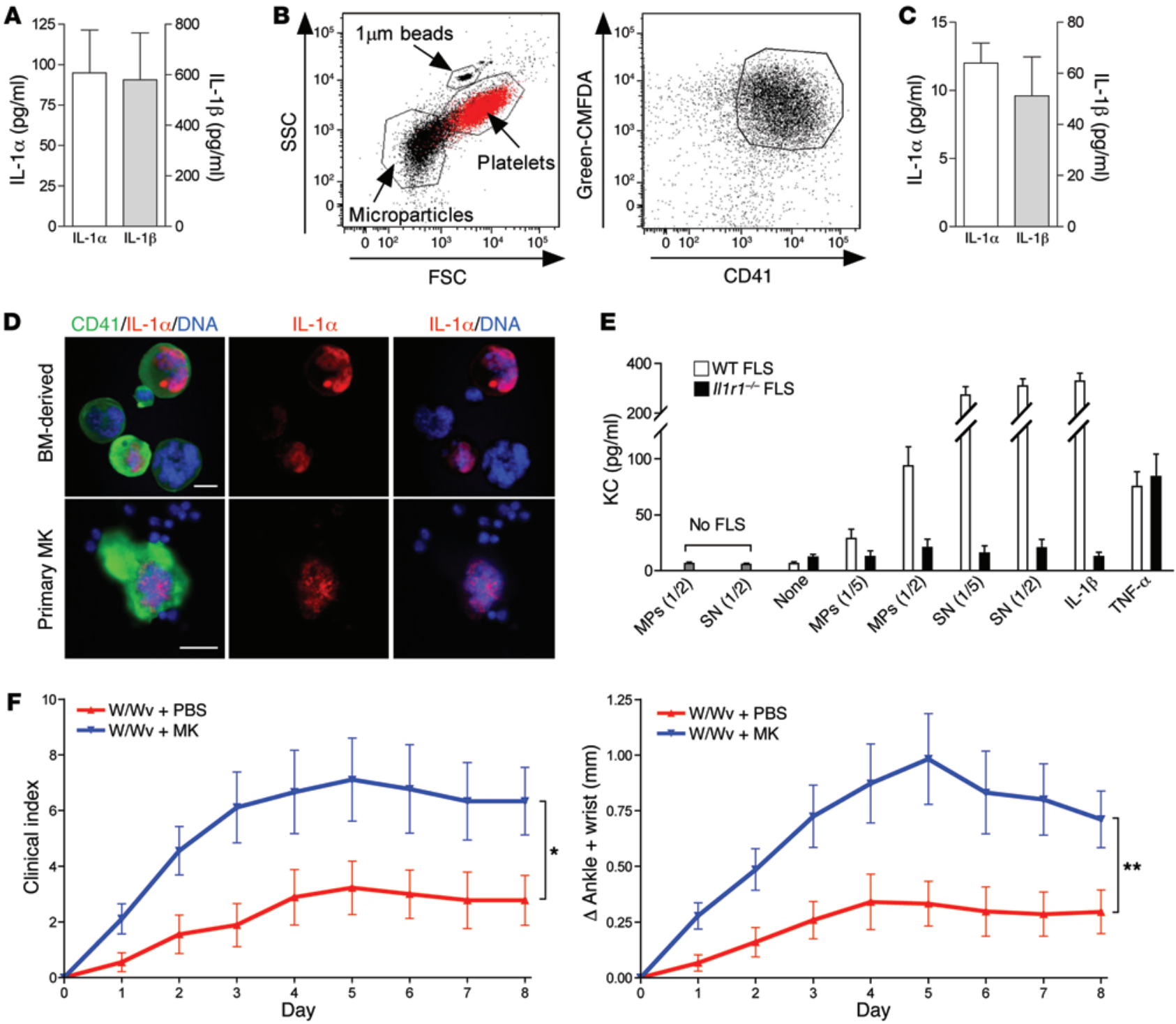

Figure 6. MKs elaborate IL-1-containing microparticles and restore arthritis susceptibility in W/Wv mice. (A) $1 \times 10^{4}$ MKs from B6 bone marrow were incubated 18 hours to test IL-1 production. Histograms show IL-1 $\alpha$ and IL-1 $\beta$ concentration in MK lysate. $n=5$. (B) Microparticle production from B6 MKs stained with green-CMFDA. Left, microparticles are gated based on size of beads (less than $1 \mu \mathrm{m}$ ). Dot plot of circulating intact platelets (red) was overlaid as a control to confirm bead sizing. Right, dot plot shows CMFDA ${ }^{+}$CD $41^{+}$events among microparticles; representative of 5 experiments. SSC, side scatter. (C) IL-1 $\alpha$ and IL-1 $\beta$ concentration in B6 MK microparticle lysates. $n=4$. (D) BM-derived MK (upper photos) and flushed BM cells (lower photos) are stained for CD41 (green) and IL-1 $\alpha$ (red). DNA is visualized with Draq5 (Blue). IL-1 $\alpha$ is detected in the nucleus of a subset of MK. Scale bars: $20 \mu \mathrm{m}$. $n=2$ per exper-

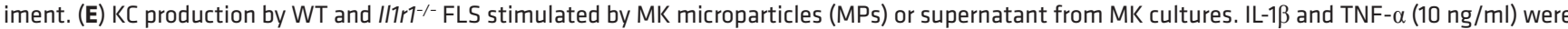
used as positive controls. $n=3$. (F) W/Wv mice were injected i.v. with PBS or $2 \times 10^{5}$ MKs. After 60 minutes, and again on day 2 , mice were treated with 150 $\mu$ li.p. K/BxN serum. Left, clinical scoring on a 0-12 scale. W/Wv injected with PBS vs. MKs, $P=0.0282$. Right, ankle and wrist thickness change. W/Wv injected PBS vs. MKs, $P=0.006 . n=9 /$ group pooled from 2 experiments. ${ }^{*} P<0.05 ;{ }^{* *} P<0.01$.

absolute number of MKs obtained per W/Wv donor was markedly lower than that from WBB6 controls (Supplemental Figure 6H). We therefore considered whether $\mathrm{W} / \mathrm{Wv}$ mice might have fewer circulating $\mathrm{CD} 41^{+}$microparticles, known to be predominantly of MK origin (36). Indeed, such a reduction was found, suggesting that a deficiency of MK microparticles might play a role in $\mathrm{W} / \mathrm{Wv}$ arthritis resistance (Figure $7 \mathrm{C}$ ).

We therefore tested whether microparticles from engrafted MKs represented a plausible contributor to arthritis restoration after adoptive transfer. Circulating donor microparticles were detected as soon as 3 hours after engraftment (Figure 7D). In agreement with published findings (36), we observed that platelet microparticles generated in vitro expressed the activation markers LAMP-1 and CD62P, while negligible levels were found on endogenous circulating $\mathrm{CD} 41^{+}$microparticles and only very low levels on those produced by MKs in vitro (Figure 7E). In our engraftment system, both endogenous circulating $\mathrm{CD} 41^{+}$microparticles and those emerging from donor MKs were CD62P- and LAMP1', strongly suggesting that newly formed microparticles have a direct MK origin and are not generated by platelets produced by engraft- 

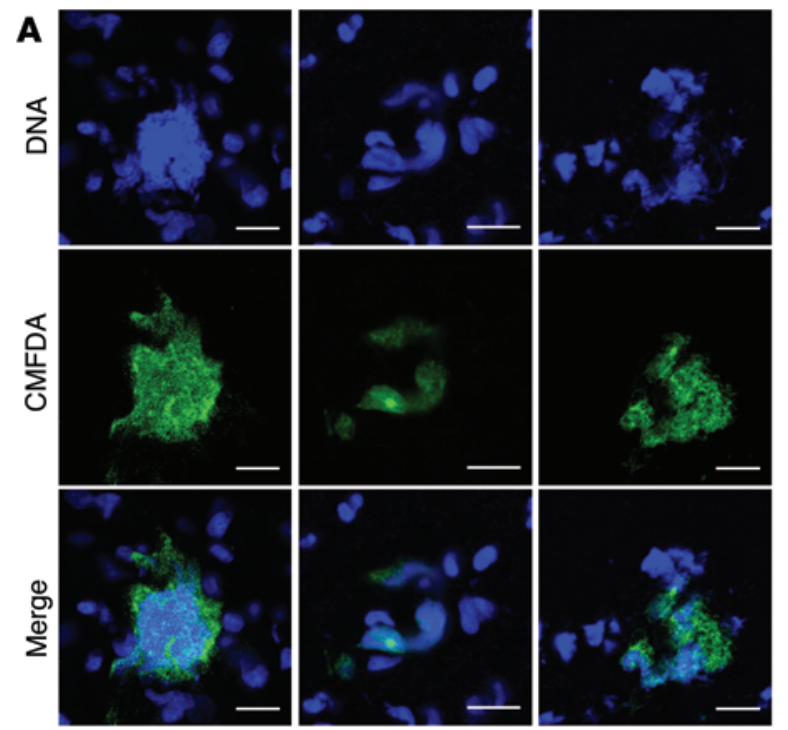

B

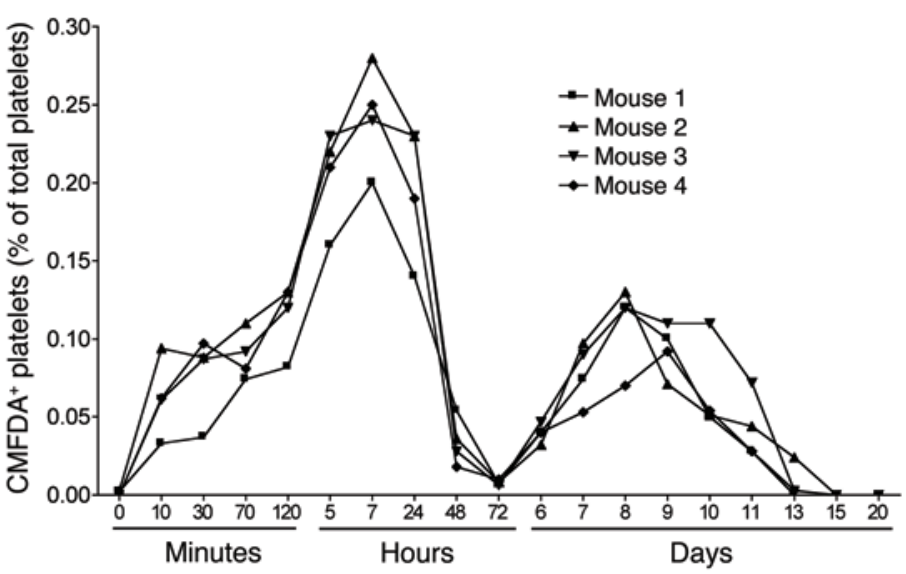

C

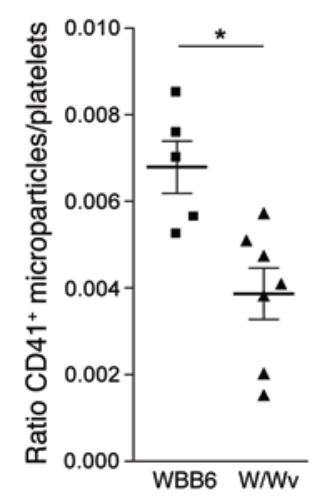

D

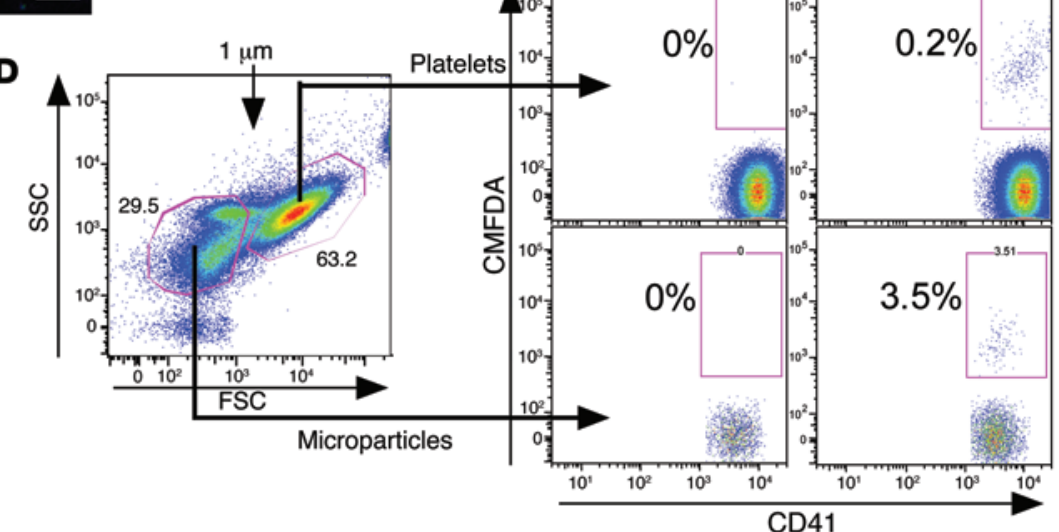

$\mathbf{E}$

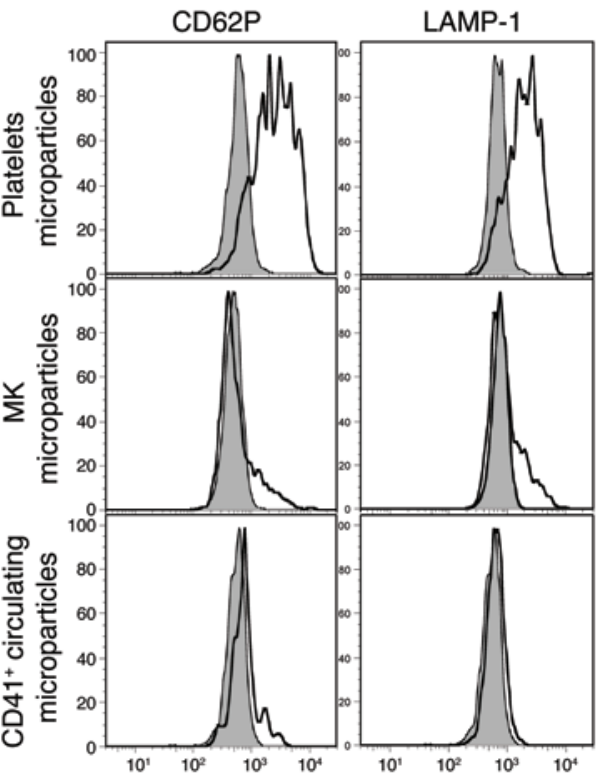

$\mathbf{F}$

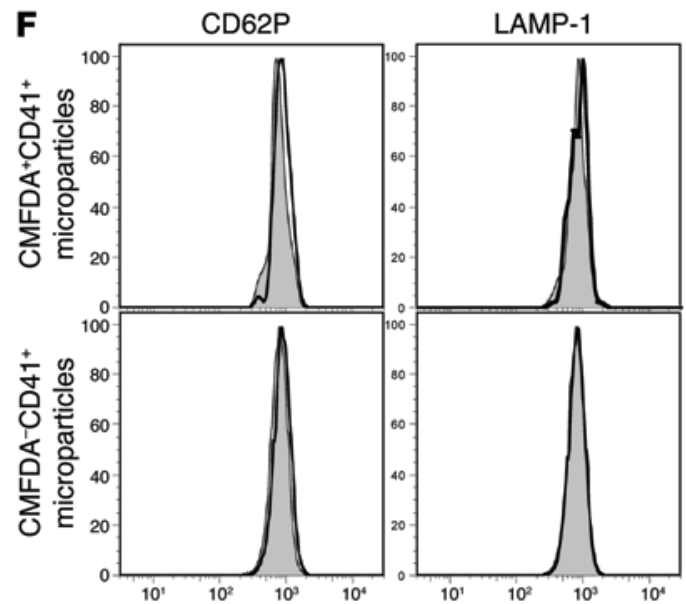

Isotype controls

Anti-CD62P/LAMP-1 mAbs

Figure 7. Injected MKs engraft in lung and produce microparticles. (A, B, D, F) W/Wv mice were injected i.v. with $2 \times 10^{5}$ MKs stained with CMFDA. (A) Detection of CMFDA ${ }^{+}$MKs in lung section 75 minutes after the engraftment. DNA was visualized with Draq5. Scale bars: $10 \mu \mathrm{m}$. Representative of 4 experiments. (B) Detection of circulating CMFDA ${ }^{+} \mathrm{CD} 41^{+}$platelets over time. Graph shows the percentages of CMFDA ${ }^{+} \mathrm{CD} 41^{+}$platelets among total CD41+ circulating platelets. (C) Detection of $C D 41^{+} \mathrm{MPs}(<1 \mu \mathrm{m})$ in unmanipulated WBB6 and W/Wv mice, normalized to total platelet number to control for dilutional artifact of cardiac aspiration directly into anticoagulant buffer. $n=5-7$ mice/group pooled from 3 experiments. ${ }^{*} P<0.05$. (D) MKs stained with CMFDA were injected in W/Wv mice as in parts $\mathbf{A}$ and $\mathbf{B}$. Detection of CMFDA+CD41+ MPs and platelets in noninjected control mice or 3 hours after MK injection. (E) CD62P and LAMP-1 expression on microparticles from activated platelets (upper histograms), MK microparticles (middle histograms), and CD41+ circulating microparticles (lower histograms). Histograms are gated on CD41+ and particle size less than $1 \mu$ m events. Representative of 2 experiments. (F) CD62P and LAMP-1 expression on the surface of circulating newly formed CD41+CMFDA ${ }^{+}$MPs (upper panels) and endogenous CD41+CMFDA- MPs (lower panels) 3 hours after CMFDA ${ }^{+}$MK injection. 
ed MKs (Figure 7F). Interestingly, exploring possible routes by which MK microparticles could be elicited, we found that a subset of both cultured and endogenous MKs expressed the high-affinity $\mathrm{Fc}$ receptor $\mathrm{Fc} \gamma \mathrm{RI}$ and that microparticle production could be enhanced by stimulation via the noncanonical Fc $\gamma$ RI ligands CRP and serum amyloid P component (SAP) (Supplemental Figure 9). Taken together, these observations show that injected MKs restore arthritis in W/Wv mice and suggest IL-1-expressing MK microparticles as one potential mechanism underlying this effect.

\section{Discussion}

Interpretation of experimental data from genetically aberrant mice is complicated by the impact of a single mutation on many aspects of a complex biological system. The use of mice with defects in the Kit-KitL axis to study the role of MCs exemplifies this problem, since Kit is expressed by both hematopoietic and nonhematopoietic cells and mutant mice demonstrate phenotypic abnormalities extending beyond MC deficiency. Recent studies have raised the concern that these abnormalities may limit the utility of Kit mutants as useful tools for experimental MC biology, calling into question multiple findings from the MC literature $(7,8,14)$.

Using IgG-mediated $\mathrm{K} / \mathrm{BxN}$ arthritis as a disease model, we undertook to evaluate the suggestion that findings generated using Kit mutants fail to accurately reflect MC function. We and others have previously used MC knockin mice to identify a role for these cells in arthritis, identifying thereby roles for MC Fc receptors, the C5a receptor, c-Jun N-terminal kinase 1, and IL-1 (11, $13,15,20)$. Our present studies confirm the lineage specificity of MC engraftment, suggesting that these studies were technically sound. However, in agreement with published results, we found that MC-deficient Wsh and Cre-Master mice remain susceptible to arthritis. What is the basis for this discrepancy?

Our data suggest a nuanced answer. Like animals lacking the MC tryptases MCP6 and MCP7 $(17,18)$, Wsh mice exhibit partial disease resistance at low doses of arthritogenic serum. We could not engraft MCs successfully into joint tissues, limiting conclusions about the role of MC deficiency in this phenotype. However, together with data from tryptase-deficient mice, the findings illustrate how partial disease resistance can be easily obscured by a strong proinflammatory stimulus. In contrast, arthritis resistance in $\mathrm{W} / \mathrm{Wv}$ - which is in any case partial, rather than absolute - reflects both hematopoietic and radioresistant (presumably stromal) compartments. Transplantation with either B6 or Wsh marrow restores arthritis, but only to the intensity permitted by the WBB6 background, while W/Wv marrow supports arthritis in either B6 or Wsh backgrounds. These data suggest a new interpretation of apparently conflicting findings among MC-deficient mice. In their capacity as immune sentinels, the role of MCs in a particular setting will vary depending on the need for amplification of the underlying inflammatory trigger. As a result, it is to be expected that the requirement for MCs will differ from one context to another. In W/Wv mice, the ability of engrafted MCs to restore susceptibility to $\mathrm{K} / \mathrm{BxN}$ arthritis accurately reflects their capacity to push this resistant system over the threshold to disease, even if MCs are dispensable in more arthritis-sensitive animals. Thus, differing findings across strains represent, not irreconcilable contradictions, but rather opportunities to understand how the pathophysiological contribution of MCs varies with context (40).
To understand the hematopoietic context that determines whether MCs play an important role in arthritis, we tested the hypothesis that neutropenia ( $30 \%$ below normal; ref. 5$)$ exposes the requirement for MCs in W/Wv mice. Indeed, transfer of neutrophils engineered to overexpress $\mathrm{Fc}$ receptors has been reported to enable arthritis (41). However, we found no evidence that WT neutrophils could do so. In further support of this observation, a 2-week marrow engraftment into unirradiated $\mathrm{W} / \mathrm{Wv}$ animals restored arthritis susceptibility even when marrow was obtained from $\mathrm{Gii}^{-/-}$mice, which are densely arthritis resistant due to an inability to produce neutrophils (26). Of note, correction of arthritis resistance by $\mathrm{Gfi1}^{-/-}$marrow proceeded even in the absence of an effect on anemia, since $\mathrm{Gfi1}^{-/-}$and W/Wv mice exhibit similar degrees of anemia (Supplemental Figure 4D). Consistent with earlier observations implicating IL-1 as a key MC mediator in arthritis $(13,15)$, expression of IL-1 by donor marrow proved essential. Surprisingly, platelet/MK-lineage factors NF-E2 and GPVI were also required. We expected that platelets would serve as the IL-1 donor, but could identify no related numeric or functional defect in $\mathrm{W} / \mathrm{Wv}$ platelets, and direct platelet transfer failed to have an impact on the phenotype. Instead, arthritis susceptibility was fully and rapidly restored by engrafted MKs, a lineage that we found capable of producing IL-1 both directly and via IL-1-containing microparticles and that (unlike platelets) is numerically deficient in W/Wv mice.

A major pitfall of engraftment experiments is inadvertent transfer of contaminating lineages. We excluded this possibility in several ways. First, we engrafted CD45.1-expressing MKs into W/Wv mice and could identify no circulating CD45.1 leukocytes. Second, we assessed tissues for engraftment of MCs and found none. Third, we performed magnetic depletion of MKs and engrafted the remaining cells, finding that loss of MKs eliminated correction of arthritis resistance. In concert with studies demonstrating pivotal roles for GPVI and NF-E2, these experiments support the hypothesis that restoration of arthritis reflects engrafted MKs.

Donor bone marrow lacking either NF-E2 or GPVI failed to restore arthritis susceptibility to W/Wv mice. Recognizing the limitation that adequate $\mathrm{NF}-\mathrm{E} 2^{-/-}$engraftment was difficult to confirm (a caveat that does not apply to the GPVI results), these observations have potentially interesting implications for MK biology. NF-E2 is a transcription factor known to regulate the formation of platelets. $\mathrm{NF}-\mathrm{E} 2^{-/-} \mathrm{MKs}$ exhibit enlarged size, paucity of granules, and failure to bind fibrinogen in response to agonists, although the role of NF-E2 in MK microparticle generation or IL-1 production is unknown $(31,42,43)$. GPVI is expressed on MKs, but its function on this lineage is undefined. In the context of our studies, it may be that this collagen/laminin receptor participates in engraftment, localization, maturation, or activation of transferred MKs. Further studies will be required to identify the mechanisms by which NF-E2 and GPVI mediate MK involvement in arthritis.

Our results do not establish the specific mechanism by which MKs mediate arthritis. Marrow transfer experiments indicate that MKs serve as direct IL-1 donors, a hypothesis confirmed by the inability of IL-1-deficient MKs to restore disease susceptibility. It is unlikely that this observation represents an artifact of a role of IL-1 in MK development because $\mathrm{Il1}^{-/-}$and WT mice demonstrate comparable concentrations of circulating CD62P-Lamp-1- MK microparticles and a similar density of MKs in bone marrow and 
spleen ( $n=5-6$ mice/group, data not shown). Provision of IL-1 could be mediated by freshly elaborated platelets, though the very low number of donor platelets ( $0.3 \%$ of the total pool, compared with $10 \%$ achieved by direct transfer) renders this possibility unlikely. MK microparticles represent a more plausible vehicle because they express IL-1, they are released by engrafted MKs, and $\mathrm{W} / \mathrm{Wv}$ mice show a deficiency in MK microparticles at baseline. Interestingly, like engrafted MKs, endogenous MKs can take up residence in the pulmonary microvasculature, migrating from bone marrow at rates estimated at up to 250,000 MKs per hour in humans (44). Residence in lung would position MKs ideally to release microparticles (and cytokines) into the systemic circulation. Intriguingly, we found that a subset of both cultured and endogenous MKs expressed Fc $\gamma \mathrm{RI}$ and that the acute-phase reactants CRP and SAP could accordingly induce enhanced microparticle production, although the role of this unexpected pathway in arthritis remains to be established. Thus, while our findings do not establish that MK microparticles mediate arthritis, they do highlight a generally unappreciated set of pathways by which MKs could participate in systemic inflammation.

It is essential to note explicitly what our data show and what they do not. We find that, in the unusual context of the Kithypomorph W/Wv mouse, MKs can mediate susceptibility to IgGmediated arthritis. This unexpected result identifies the MKs as a lineage of interest in systemic inflammatory disease and highlights the potential immunological significance of little-understood aspects of MK physiology, including release of circulating microparticles, migration to lung, and expression of $\mathrm{Fc}$ receptors. Our data do not address whether MKs play a similar role in the otherwise intact host. Exploration of this biology will require development of new tools to segregate the immune functions of MKs from their role to protect the host from lethal hemorrhage.

Taken together, the findings presented here have broad implications. First, they reconcile conflicting studies in the MC literature by illustrating how the role of MCs in arthritis varies with disease trigger and host context. Second, they identify MKs as a participant in IL-1-mediated systemic inflammatory disease, raising the intriguing possibility that MKs could contribute in other contexts and identifying MKs as an important lineage for further immunological research.

\section{Methods}

Mice. C57BL/6, CD45.1 B6 (B6.SJL-Ptprca Pepc ${ }^{b}$ /BoyJ), W/Wv (WBB6 F1-Kit $\left.{ }^{w} / K^{W}{ }^{W-v}\right)$, and Il1r1 $1^{--}\left(\right.$B6.129S7-Il1r1 $\left.{ }^{\text {tmIImx }} / \mathrm{J}\right)$ mice were purchased from The Jackson Laboratory. C57BL/6-Kit ${ }^{\text {Wsh } / \text { wsh }}$ (Wsh) mice were maintained at Jackson Laboratory (45). Gp1ba/- mice were generated as described (30). $I l 1 \mathrm{a}^{-/-} \mathrm{Il} l \mathrm{~b}^{-/-}$mice were a gift of Yoichiro Iwakura (46). $\mathrm{Gfi1}^{-/-}$mice were a gift of Hanno Hock (Massachusetts General Hospital, Boston, MA, USA) (27). Gpvi־- mice were derived as described (47). $\mathrm{NF}-\mathrm{E}^{-/-}$mice were a gift of Ramesh Shivdasani (Dana-Farber Cancer Institute, Boston, MA, USA) (31). EGFP-CD41 mice were a gift of Thomas Graf (Institucio Catalana de Recerca i Estudis Avancat, Barcelona, Spain) (39). Cre-Master mice were a gift of Hans-Reimer Rodewald (German Cancer Research Center, Heidelberg, Germany) (7). All experiments employed male mice aged 8 to 10 weeks unless otherwise specified.

Serum transfer and arthritis scoring. High doses $(150 \mu \mathrm{l})$ or low doses $(50 \mu \mathrm{l})$ of pooled serum from 9- to 11 -week-old $\mathrm{K} / \mathrm{BxN}$ mice were transferred i.p. on day 0 and day 2. Arthritis was graded using a 0 to 12 clinical scale (0 to 3 per paw) as described (11), as well as by caliper of wrists and ankles. Histological assessment of ankles was performed on paraffin-embedded sections stained with H\&E. Synovial inflammation, cartilage injury, and bone erosion were graded on a 0 to 5 scale as described (15).

Bone marrow transplantation. To study the role for hematopoietic and radioresistant compartments in arthritis, recipient mice were lethally irradiated ( 5.5 , then $5 \mathrm{~Gy}$ ), followed by immediate rescue with donor femoral and tibial bone marrow (1 donor per recipient). Transplanted animals were maintained on water supplemented with enrofloxacin through full engraftment by 8 weeks, followed by initiation of arthritis. In other bone marrow transfer experiments, unirradiated $\mathrm{W} / \mathrm{Wv}$ mice were engrafted with bone marrow (1 donor per 2 recipients) and arthritis was initiated after 14 days.

$M K$ generation and adoptive transfer. Bone marrow cells were cultured in complete RPMI medium supplemented with $1 \%$ supernatant from the thrombopoietin-producing (TPO-producing) fibroblast cell line GP122 (48). Of note, IL- $1 \alpha$ and IL-1 $\beta$ were undetectable in the supernatant of the GP122 cell line (data not shown). After 4 days, MKs were separated from bone marrow cells using a 2-step albumin gradient as described (49). For engraftment, $2 \times 10^{5} \mathrm{MKs}$ in $200 \mu \mathrm{PBS}$ were injected by tail vein. In some experiments, enriched MKs were stained with $5 \mu \mathrm{M}$ green-CMFDA (Life Technologies) according to manufacturer protocol. In other experiments, contaminating CD41- cells were magnetically separated from enriched MKs using magnetic separation columns (Miltenyi Biotech).

Platelet isolation and adoptive transfer. Platelet isolation and transfer were done as described (33). Briefly, whole blood was drawn into a syringe containing acid citrate dextrose anticoagulant $(0.085 \mathrm{M}$ sodium citrate, $0.0702 \mathrm{M}$ citric acid, $0.111 \mathrm{M}$ dextrose, $\mathrm{pH} 4.5$ ) and transferred into 1.5-ml Eppendorf tubes containing $350 \mu \mathrm{l}$ of $\mathrm{Ca}^{2+}$ free Tyrode's buffer (134 mM NaCl, $2.9 \mathrm{mM} \mathrm{KCl}, 0.34 \mathrm{mM} \mathrm{Na}_{2} \mathrm{HPO}_{4}, 12$ $\mathrm{mM} \mathrm{NaHCO}_{3}, 20 \mathrm{mM}$ HEPES, $1 \mathrm{mM} \mathrm{MgCl}, 5 \mathrm{mM}$ glucose, $0.5 \mathrm{mg} / \mathrm{ml}$ $\mathrm{BSA}, \mathrm{pH}$ 6.5). Following mixing via gentle inversion, larger cells were pelleted via a single centrifugation at $600 \mathrm{~g}$ for 3 minutes at room temperature (RT) and a second centrifugation of supernatant at $400 \mathrm{~g}$ for 2 minutes to obtain platelet-rich plasma (PRP). Platelet-poor plasma (PPP) was obtained from PRP by centrifugation twice at $1,300 \mathrm{~g}$ for 5 minutes to pellet all platelets, and pellets were resuspended with PRP to obtain a high-concentration PRP. PRP or PPP was administered i.v. daily for 5 days ( 5 donors per recipient per day).

Platelet activation and microparticle isolation. Pellets were isolated from PRP, obtained as above, via centrifugation at 1,300 $g$ for 5 minutes with rapid resuspension of the pellet into $50 \mu \mathrm{l}$ of Tyrode's buffer. Platelets were resuspended in $500 \mu \mathrm{DMEM}$ (containing calcium) and stimulated at RT with $1 \mu \mathrm{g} / \mathrm{ml}$ crosslinked collagen-related peptide (xlCRP, GPVI ligand, obtained from Richard Farndale, University of Cambridge, Cambridge, United Kingdom), and $30 \mu$ aliquots were harvested serially to assess by flow cytometry the proportion of CD $41^{+}$ events within the usual platelet scatter gate. At 24 hours, the remaining supernatant was harvested and cleared of the remaining platelets by centrifugation at $1,300 \mathrm{~g}$ for 5 minutes; microparticles were purified by ultracentrifugation at $21,000 \mathrm{~g}$ for 1.5 hours at $18^{\circ} \mathrm{C}$. To isolate MK microparticles, supernatant from MKs cultured for 18 hours were centrifuged at $500 \mathrm{~g}$ for 5 minutes and $1,300 \mathrm{~g}$ for 5 minutes, then ultracentrifuged at $21,000 \mathrm{~g}$ for 1.5 hours. 
Neutrophil transfer. Bone marrow neutrophils were enriched by gradient centrifugation as published (25) and transferred i.v. once a day on days 0 through 4 (2 donors/d/recipient).

FLS generation and stimulation. WT and Illr1 $1^{-/}$FLS were obtained from collagenase digest of isolated ankles as described (29) and grown in complete FLS culture media containing L-glutamine, $2 \beta$ mercaptoethanol, and essential and nonessential amino acids for at least 3 passages. 20,000 FLS were put in 96-well plates for 2 days, washed, and stimulated for 14 hours with microparticles or supernatants from MKs or activated platelets for KC production.

ELISA assays. Murine IL- $1 \alpha, \mathrm{KC}$ (Peprotech), IL-1 $\beta$ (BioLegend), IL-6 (R\&D Systems), CRP, and serum amyloid A (Immunology Consultants Laboratory) were quantified using commercial ELISA assays from the indicated providers.

Flow cytometry. Flow cytometry was performed on a BD FACSCantoII flow cytometer. Blood stains were performed in whole blood diluted 1:1 with PBS/5\% FBS flow cytometry buffer, followed by 2 rounds of red blood cell lysis in ACK buffer. Spleen was disaggregated by manual pressure through a $100-\mu \mathrm{m}$ mesh, and femoral flush bone marrow was similarly filtered before blocking and staining in flow cytometry buffer. Antibodies used were anti-CD41 (clone MWReg30), anti-FceR1 (clone MAR-1, eBioscience); anti-CD62P (clone RB40.34, BD Bioscience); anti-CD3 (clone 145-2C11), anti-CD11c (clone N418), anti-CD19 (clone 6D5), anti-CD45.1 (clone A20), anti-CD45.2 (clone 104), anti-CD61 (clone 2C9.G2), anti-Gr1 (clone RB6-8C5), anti-F4/80 (clone BM8), anti-LAMP-1 (clone 1D4B), anti-cKit (clone 2B8, BioLegend); and anti-GPVI (clone JAQ1, emfret ANALYTICS).

Microscopy. MK engraftment into lung was as follows: immediately after excision, lungs were embedded in OCT, frozen in dry ice filled with 2-methyl butane, and cryosected ( $6 \mu \mathrm{m}$ in thickness). Sections were fixed for 15 minutes with $4 \%$ PFA, washed, and incubated with PBS containing $5 \mu \mathrm{M}$ Draq5 (eBioscience). After washing, sections were mounted with FluorSave reagent (Calbiochem). IL-1 $\alpha$ and Fc $\gamma$ R1 expression in MK were as follows: bone marrow-derived MKs or flushed bone marrow cells were fixed in 2\% PFA for 30 minutes at RT. After washing, cells were suspended in PBS containing 3\% FCS supplemented or not with $0.1 \%$ saponin and incubated overnight at $4^{\circ} \mathrm{C}$ with IL-1 $\alpha$ (clone 166926, R\&D) or anti-CD64 (clone X54-5/7.1, BioLegend; clone 290322, R\&D; or rabbit polyclonal, SinoBiological Inc.). After washing, secondary antibodies diluted to 1:200 were added for 1 hour. After washing, cells were incubated with $10 \mu \mathrm{g} / \mathrm{ml}$ antiCD41 (clone MWReg30) for 1 hour. Draq5 or Hoechst (Thermo Fisher Scientific) was added for the last 15 minutes before washing with PBS, followed by Cytospin. Cells were mounted on slides using FluorSave reagent. Samples were observed using Nikon C1 Plus Confocal Laser Scanner or a Zeiss LSM 710 Multiphoton Laser Scanner.

For additional information, see Supplemental Methods.

Statistics. Unless otherwise stated, comparisons between 2 conditions employed the 2-tailed Wilcoxon matched pairs test and comparisons between 2 groups of mice over time employed the 2-tailed Mann-Whitney $U$ test. For measurement and scoring of arthritis over time, we used 2-way ANOVA followed by Bonferroni's post-test. Statistical analyses were performed with GraphPad Prism software. $P<0.05$ was considered significant.

Study approval. All experiments were approved by the Institutional Animal Care and Use Committee of the Dana Farber Cancer Institute (Boston, Massachusetts, USA; protocol 03-028). All mice were maintained under specific pathogen-free conditions.

\section{Author contributions}

PC and PAN conceptualized and designed the research and wrote the manuscript. PC, LRP, TJ, MHC, MMC, IM, SL, MFG, EB, and PAN acquired data. PC and PAN analyzed data. JNT, YI, JW, PAM, and JEI provided samples, reagents, and other project assistance. All authors revised the manuscript. PAN provided overall project guidance and supervision.

\section{Acknowledgments}

We are grateful to Hans-Reimer Rodewald and Thorsten Feyerabend for their thoughtful discussion of the manuscript. This work was supported by NIH grants R01H168130 (to JEI) and R21AR062328, R01AR065538, and P30AR070253 (to PAN), and by the Cogan Family Foundation and the Fundación Bechara (to PAN).

Address correspondence to: Peter A. Nigrovic, Brigham and Women's Hospital, Division of Rheumatology, Immunology and Allergy, Building for Transformative Medicine, Room 06002L, 60 Fenwood Road, Boston, Massachusetts 02115, USA. Phone: 617.525.1031; E-mail:pnigrovic@bwh.harvard.edu.

LRP's present address is: Department of Internal Medicine, Division of Pulmonary and Critical Care Medicine, University of Michigan, Ann Arbor, Michigan, USA.

MMC's present address is: Takeda Pharmaceuticals International Inc., Deerfield, Illinois, USA.
1. Ehrlich P. Beiträge zur Theorie und Praxis der histologischen Färbung [doctoral thesis]. Leipzig, Germany: Leipzig University; 1878.

2. Wong GW, Zhuo L, Kimata K, Lam BK, Satoh N, Stevens RL. Ancient origin of mast cells. Biochem Biophys Res Commun. 2014;451(2):314-318.

3. Katz HR, Austen KF. Mast cell deficiency, a game of kit and mouse. Immunity. 2011;35(5):668-670.

4. Galli SJ, Kitamura Y. Genetically mast-celldeficient $\mathrm{W} / \mathrm{Wv}$ and $\mathrm{Sl} / \mathrm{Sld}$ mice. Their value for the analysis of the roles of mast cells in biologic responses in vivo. Am J Pathol. 1987;127(1):191-198.

5. Nigrovic PA, et al. Genetic inversion in mast cell-deficient (Wsh) mice interrupts corin and manifests as hematopoietic and cardiac aberrancy. Am J Pathol. 2008;173(6):1693-1701.

6. Nakano T, et al. Fate of bone marrow-derived cultured mast cells after intracutaneous, intraperitoneal, and intravenous transfer into genetically mast cell-deficient W/Wv mice. Evidence that cultured mast cells can give rise to both connective tissue type and mucosal mast cells. J Exp Med.1985;162(3):1025-1043.

7. Feyerabend TB, et al. Cre-mediated cell ablation contests mast cell contribution in models of antibody- and T cell-mediated autoimmunity. Immunity. 2011;35(5):832-844.

8. Dudeck A, et al. Mast cells are key promoters of contact allergy that mediate the adjuvant effects of haptens. Immunity. 2011;34(6):973-984.

9. Scholten J, et al. Mast cell-specific Cre/loxPmediated recombination in vivo. Transgenic Res. 2008;17(2):307-315.

10. Grimbaldeston MA, Nakae S, Kalesnikoff J, Tsai M, Galli SJ. Mast cell-derived interleukin 10 limits skin pathology in contact dermatitis and chronic irradiation with ultraviolet B. Nat Immunol. 2007;8(10):1095-1104.

11. Lee DM, Friend DS, Gurish MF, Benoist C, Mathis D, Brenner MB. Mast cells: a cellular link between autoantibodies and inflammatory arthritis. Science. 2002;297(5587):1689-1692. 
12. Corr M, Crain B. The role of FcgammaR signaling in the K/B x N serum transfer model of arthritis. J Immunol. 2002;169(11):6604-6609.

13. Guma M, et al. JNK1 controls mast cell degranulation and IL-1\{beta\} production in inflammatory arthritis. Proc Natl Acad Sci U S A. 2010;107(51):22122-22127.

14. Zhou JS, Xing W, Friend DS, Austen KF, Katz HR. Mast cell deficiency in Kit(W-sh) mice does not impair antibody-mediated arthritis. JExp Med. 2007;204(12):2797-2802

15. Nigrovic PA, et al. Mast cells contribute to initiation of autoantibody-mediated arthritis via IL-1. Proc Natl Acad Sci USA. 2007;104(7):2325-2330.

16. Schubert N, et al. Mast cell promotion of $\mathrm{T}$ cell-driven antigen-induced arthritis despite being dispensable for antibody-induced arthritis in which T cells are bypassed. Arthritis Rheuma tol. 2015;67(4):903-913

17. McNeil HP, et al. The mouse mast cell-restricted tetramer-forming tryptases mouse mast cell protease 6 and mouse mast cell protease 7 are critical mediators in inflammatory arthritis. Arthritis Rheum. 2008;58(8):2338-2346.

18. Shin K, et al. Mast cells contribute to autoimmune inflammatory arthritis via their tryptase/heparin complexes. JImmunol. 2009;182(1):647-656.

19. Binstadt BA, et al. Particularities of the vasculature can promote the organ specificity of autoimmune attack. Nat Immunol. 2006;7(3):284-292.

20. Nigrovic PA, et al. C5a receptor enables participation of mast cells in immune complex arthritis independently of Fc $\gamma$ receptor modulation. Arthritis Rheum. 2010;62(11):3322-3333.

21. Lyon MF, Glenister PH. A new allele sash (Wsh) at the $\mathrm{W}$-locus and a spontaneous recessive lethal in mice. Genet Res. 1982;39(3):315-322.

22. Wipke BT, Allen PM. Essential role of neutrophils in the initiation and progression of a murine model of rheumatoid arthritis. J Immunol. 2001;167(3):1601-1608

23. Wang JX, et al. Ly6G ligation blocks recruitment of neutrophils via a $\beta 2$-integrin-dependent mechanism. Blood. 2012;120(7):1489-1498.

24. Chou RC, et al. Lipid-cytokine-chemokine cascade drives neutrophil recruitment in a murine model of inflammatory arthritis. Immunity. 2010;33(2):266-278

25. Chen M, et al. Neutrophil-derived leukotriene B4 is required for inflammatory arthritis. JExp Med. 2006;203(4):837-842.

26. Monach PA, et al. Neutrophils in a mouse model of autoantibody-mediated arthritis: critical producers of Fc receptor gamma, the receptor for C5a, and lymphocyte function-associated antigen 1. Arthritis Rheum. 2010;62(3):753-764.

27. Hock $\mathrm{H}$, et al. Intrinsic requirement for zinc finger transcription factor Gfi-1 in neutrophil differentiation. Immunity. 2003;18(1):109-120.

28. Ebbe S, Carpenter D, Yee T. Megakaryocytopenia in W/Wv mice is accompanied by an increase in size within ploidy groups and acceleration of maturation. Blood. 1989;74(1):94-98.

29. Boilard E, et al. Platelets amplify inflammation in arthritis via collagen-dependent microparticle production. Science. 2010;327(5965):580-583.

30. Ware J, Russell S, Ruggeri ZM. Generation and rescue of a murine model of platelet dysfunction: the Bernard-Soulier syndrome. Proc Natl Acad Sci US A. 2000;97(6):2803-2808.

31. Shivdasani RA, et al. Transcription factor NF-E2 is required for platelet formation independent of the actions of thrombopoietin/ MGDF in megakaryocyte development. Cell. 1995;81(5):695-704.

32. Jandrot-Perrus $\mathrm{M}$, et al. Cloning, characterization, and functional studies of human and mouse glycoprotein VI: a platelet-specific collagen receptor from the immunoglobulin superfamily. Blood. 2000;96(5):1798-1807.

33. Boilard E, et al. Platelets participate in synovitis via Cox-1-dependent synthesis of prostacyclin independently of microparticle generation. J Immunol. 2011;186(7):4361-4366.

34. Grabarek J, et al. Human kit ligand (stem cell factor) modulates platelet activation in vitro. J Biol Chem. 1994;269(34):21718-21724.

35. Avraham H, et al. Effects of the stem cell factor, c-kit ligand, on human megakaryocytic cells. Blood.1992;79(2):365-371.

36. Flaumenhaft R, et al. Megakaryocyte-derived microparticles: direct visualization and distinction from platelet-derived microparticles. Blood. 2009;113(5):1112-1121.

37. Fuentes $\mathrm{R}$, et al. Infusion of mature megakaryocytes into mice yields functional platelets. J Clin Invest. 2010;120(11):3917-3922.

38. Mazharian A, Watson SP, Séverin S. Critical role for ERK1/2 in bone marrow and fetal liverderived primary megakaryocyte differentiation, motility, and proplatelet formation. Exp Hematol. 2009;37(10):1238-1249.e5.

39. Zhang J, Varas F, Stadtfeld M, Heck S, Faust N, Graf T. CD41-YFP mice allow in vivo labeling of megakaryocytic cells and reveal a subset of platelets hyperreactive to thrombin stimulation. Exp Hematol. 2007;35(3):490-499.

40. Reber LL, Marichal T, Galli SJ. New models for analyzing mast cell functions in vivo. Trends Immunol. 2012;33(12):613-625.

41. Tsuboi N, et al. Regulation of human neutrophil Fc $\gamma$ receptor IIa by C5a receptor promotes inflammatory arthritis in mice. Arthritis Rheum. 2011;63(2):467-478.

42. Lecine P, Villeval JL, Vyas P, Swencki B, Xu Y, Shivdasani RA. Mice lacking transcription factor NF-E2 provide in vivo validation of the proplatelet model of thrombocytopoiesis and show a platelet production defect that is intrinsic to megakaryocytes. Blood.1998;92(5):1608-1616.

43. Shiraga M, et al. Primary megakaryocytes reveal a role for transcription factor NF-E2 in integrin alpha IIb beta 3 signaling. J Cell Biol. 1999;147(7):1419-1430.

44. Weyrich AS, Zimmerman GA. Platelets in lung biology. Annu Rev Physiol. 2013;75:569-591.

45. Wolters PJ, et al. Tissue-selective mast cell reconstitution and differential lung gene expression in mast cell-deficient Kit(W-sh)/Kit(W-sh) sash mice. Clin Exp Allergy. 2005;35(1):82-88.

46. Horai R, et al. Production of mice deficient in genes for interleukin (IL)-1alpha, IL-1beta, IL-1alpha/beta, and IL-1 receptor antagonist shows that IL-1beta is crucial in turpentine-induced fever development and glucocorticoid secretion. JExp Med.1998;187(9):1463-1475.

47. Kato K, et al. The contribution of glycoprotein V to stable platelet adhesion and thrombus formation illustrated by targeted gene deletion. Blood 2003;102(5):1701-1707.

48. Villeval JL, et al. High thrombopoietin production by hematopoietic cells induces a fatal myeloproliferative syndrome in mice. Blood. 1997;90(11):4369-4383.

49. Shivdasani RA, Schulze H. Culture, expansion, and differentiation of murine megakaryocytes. Curr Protoc Immunol. 2005; Chapter 22:Unit 22F.6. 